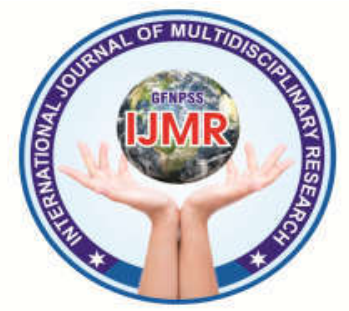

\title{
A STUDY TO ASSESS THE EFFECTIVENESS OF INSTRUCTIONAL MODULE REGARDING EFFECTS OF TELEVISION WATCHING ON BEHAVIOUR AMONG SCHOOL AGE CHILDREN IN SELECTED SCHOOL AT SIKAR CITY
}

\author{
DR. ABDUL LATIF
}

\section{(Principal Khwaja Gharib Nawaz College of Nursing) \\ Corresponding Email: drabdullatif20@gmail.com}

\begin{abstract}
Introduction: Television has captured the hearts and minds of the people. Its expansion has been much more dramatic than that of radio, or automobile. It has became important part of our day to day life, so much so it is difficult to say that whether it is luxury or necessity for most of the people.
\end{abstract}

Material and Methods- quantitative approach, one group pre test post test research design, study setting: urban area prince academy, sample size: 300 school age children, sampling technique: simple random sampling technique, data collection instrument: structured interview schedule with questionnaire was used for study.

Result: Classification of frequencies and percentages for the analyze of demographic data Calculation of frequencies and percentages for the analyze the existing level of knowledge Computation of mean and standard deviation of pre-test and post-test scores Computation of wilcoxon Signed ranks test to compare difference in mean knowledge for pre-test and posttest Computation of Chi Square test to evaluate whether the difference is statistically significant for the parameters of the study.

Conclusion: The study concluded that IM on effects of television watching on behaviour among school age children was an effective method to improve their knowledge and help them to adapt to preventive measure of ill effects of television watching on behaviour and enable them to live a better quality of life.

Keyword: HDL, DVD, AAP, CTV, NHANES 


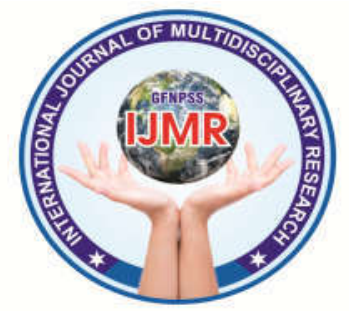

\section{INTRODUCTION:}

It has proved to be a single most pervasive social phenomenon of $20^{\text {th }}$ century. So, television cannot be ignored anymore. It is a inseparable part of a family where is a powerful visual medium of information stimulation, discussion, pleasure, and profit

\section{METHODOLOGY}

The conceptual frame work selected for the study was based on “General System Theory". It was proposed by Ludwig Von Bertalanffy in (1968). The conceptual frame work of the theory includes input, throughput and output.

In view of the nature of the problem selected and objective to be accomplished, Quasi- experimental design was selected; one group pre test post test design was considered most suitable for the study, "A study to assess the effectiveness of instructional module regarding effects of television watching on behaviour among school age children in selected school.

Simple random sampling technique was used and 300 school age children were assessed from selected in Prince Academy at Sikar. The method used for the data collection is interview schedule by using structured knowledge questionnaire to assess the knowledge regarding effects of television watching on behaviour. Instructional Module on effects of television watching on behaviour was developed.

A pre test was conducted by administering interview schedule with structured knowledge questionnaire to the samples after obtaining consent; on the same day Instructional Module was administered and the post-test was conducted by using the same structured knowledge questionnaire after the $10^{\text {th }}$ day of administration of Instructional Module.

Data was collected from 05-122015 to 20-01-2016. The obtained data was analyzed by using descriptive and inferential statistics, in terms of frequency, percentage, mean median, standard deviation, wilcoxon signed ranks-test and chi-square test.

\section{Association between the post test}

knowledge scores with selected

demographic variables 


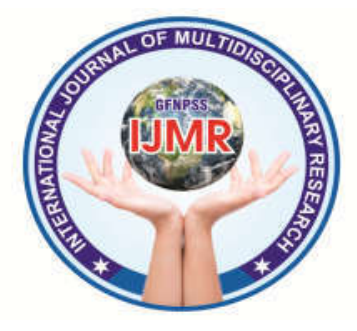

\begin{tabular}{|c|c|c|c|c|c|c|}
\hline \multirow{3}{*}{$\begin{array}{l}\text { S. } \\
\text { N. }\end{array}$} & \multirow{3}{*}{$\begin{array}{l}\text { Demogra } \\
\text { phic data }\end{array}$} & \multirow{3}{*}{ Categories } & \multicolumn{4}{|c|}{ Overall Knowledge } \\
\hline & & & $\begin{array}{l}\text { Inade } \\
\text { quate } \\
(<50)\end{array}$ & $\begin{array}{c}\text { Moder } \\
\text { ate(51- } \\
70)\end{array}$ & $\begin{array}{l}\text { Adequ } \\
\text { ate }(>7 \\
\text { 0) }\end{array}$ & $\begin{array}{l}\chi^{2} \\
\text { Value }\end{array}$ \\
\hline & & & $\mathbf{F}$ & $\mathbf{F}$ & $\mathbf{F}$ & \\
\hline \multirow[t]{3}{*}{1.} & \multirow{3}{*}{$\begin{array}{l}\text { Age of } \\
\text { Child }\end{array}$} & $12-13$ yrs. & 2 & 23 & 70 & \multirow{3}{*}{$\begin{array}{l}3.52 \\
\mathrm{DF}=4 \\
\mathrm{NS}\end{array}$} \\
\hline & & $14-15$ yrs. & 2 & 37 & 81 & \\
\hline & & $16-17$ yrs. & 1 & 17 & 67 & \\
\hline \multirow[t]{2}{*}{2.} & \multirow[t]{2}{*}{$\begin{array}{l}\text { Class of } \\
\text { Child }\end{array}$} & $\begin{array}{l}7-9 \\
\text { stranded }\end{array}$ & 4 & 51 & 135 & \multirow{2}{*}{$\begin{array}{l}1.063 \\
D F=2 \\
S\end{array}$} \\
\hline & & $\begin{array}{l}10-12 \\
\text { Stranded }\end{array}$ & 1 & 26 & 83 & \\
\hline \multirow[t]{2}{*}{3.} & \multirow[t]{2}{*}{ Sex } & Male & 5 & 49 & 126 & \multirow{2}{*}{$\begin{array}{l}4.198 \\
\mathrm{DF}=2 \\
\mathrm{NS}\end{array}$} \\
\hline & & Female & 0 & 28 & 92 & \\
\hline \multirow[t]{4}{*}{4.} & \multirow{4}{*}{$\begin{array}{l}\text { Monthly } \\
\text { family } \\
\text { income } \\
\text { (in Rs./-) }\end{array}$} & $\begin{array}{l}\text { Below } \\
5000 /-\end{array}$ & 0 & 20 & 50 & \multirow{4}{*}{$\begin{array}{l}8.398 \\
\mathrm{DF}=6 \\
\mathrm{NS}\end{array}$} \\
\hline & & $\begin{array}{l}5001- \\
6000 /-\end{array}$ & 5 & 27 & 88 & \\
\hline & & $\begin{array}{l}6001- \\
7000 /-\end{array}$ & 0 & 25 & 65 & \\
\hline & & $\begin{array}{l}\text { Above } \\
7000 /-\end{array}$ & 0 & 5 & 15 & \\
\hline \multirow[t]{4}{*}{5.} & \multirow{4}{*}{$\begin{array}{l}\text { Hours of } \\
\text { watching } \\
\text { televisio } \\
\text { n daily }\end{array}$} & 2 Hrs. & 2 & 20 & 68 & \multirow{4}{*}{$\begin{array}{l}2.899 \\
\mathrm{DF}= \\
6 \\
\mathrm{NS}\end{array}$} \\
\hline & & 3-5 Hrs. & 2 & 46 & 112 & \\
\hline & & 5-7 Hrs. & 1 & 7 & 22 & \\
\hline & & $\begin{array}{l}\text { Above } 7 \\
\text { Hrs. }\end{array}$ & 0 & 4 & 16 & \\
\hline \multirow[t]{4}{*}{6.} & \multirow{4}{*}{$\begin{array}{l}\text { Siblings } \\
\text { in family }\end{array}$} & One & 1 & 23 & 71 & \multirow{4}{*}{$\begin{array}{l}1.969 \\
\mathrm{DF}=6 \\
\mathrm{NS}\end{array}$} \\
\hline & & Two & 4 & 35 & 101 & \\
\hline & & $\begin{array}{l}\text { More than } \\
\text { two }\end{array}$ & 0 & 12 & 28 & \\
\hline & & $\begin{array}{l}\text { No } \\
\text { siblings }\end{array}$ & 0 & 7 & 18 & \\
\hline \multirow[t]{3}{*}{7.} & \multirow{3}{*}{$\begin{array}{l}\text { Snacks } \\
\text { between } \\
\text { televisio } \\
\mathrm{n} \\
\text { watching }\end{array}$} & $\begin{array}{l}\text { Traditiona } \\
1 \text { Home } \\
\text { made }\end{array}$ & 2 & 22 & 61 & \multirow{3}{*}{$\begin{array}{l}0.507 \\
\mathrm{DF}=4 \\
\mathrm{NS}\end{array}$} \\
\hline & & $\begin{array}{l}\text { Commerci } \\
\text { al aid } \\
\text { foods }\end{array}$ & 2 & 42 & 121 & \\
\hline & & $\begin{array}{l}\text { Avoiding } \\
\text { food }\end{array}$ & 1 & 13 & 36 & \\
\hline \multirow[t]{3}{*}{8.} & \multirow{3}{*}{$\begin{array}{l}\text { Minimum } \\
\text { distance } \\
\text { kept } \\
\text { while } \\
\text { watching } \\
\text { television }\end{array}$} & $\begin{array}{l}\text { Less than } \\
4 \text { feet }\end{array}$ & 1 & 11 & 33 & \multirow{3}{*}{$\begin{array}{l}2.686 \\
\mathrm{DF}=4 \\
\mathrm{NS}\end{array}$} \\
\hline & & 4-6 feet & 4 & 42 & 129 & \\
\hline & & $\begin{array}{l}\text { More than } \\
6 \text { feet }\end{array}$ & 0 & 24 & 56 & \\
\hline \multirow[t]{4}{*}{9.} & \multirow{4}{*}{$\begin{array}{l}\text { Types of } \\
\text { TV } \\
\text { program } \\
\text { mes } \\
\text { watching }\end{array}$} & $\begin{array}{l}\text { Education } \\
\text { al }\end{array}$ & 0 & 13 & 32 & $\begin{array}{l}4.773 \\
D F=6\end{array}$ \\
\hline & & Frictional & 5 & 33 & 107 & \\
\hline & & Social & 0 & 24 & 56 & \\
\hline & & Mixed & 0 & 7 & 23 & \\
\hline
\end{tabular}

Key:

$>$ NS - Not significant at level of $>0.05$

$>$ S- Significant at level of $>0.01$

DF: degrees of freedom

$>$ F-Frequency

$>$ \%-Percentage

$>\chi^{2}$ - Chi square.

\section{RESULTS}

The measured findings indicated that school age children had inadequate knowledge in various aspects of prevention of ill effects of television watching on behaviour. IM was proved to be a very effective means of providing information regarding effects of television watching on behaviour. The mean of post test knowledge score is significantly higher than the mean of pre test knowledge score $\quad($ at $p<0.05$ level $)$ and there was no significant association between post test knowledge score with their selected demographic variables as the analysis showed that the chi-square calculated value was less than the chisquare table value except class of child. 


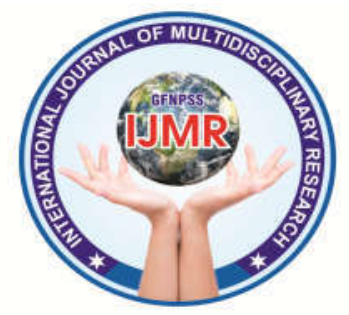

\section{INTERPRETATION AND}

\section{CONCLUSION}

On the basis of findings, it is recommended that a similar study may be done adolescence. It is recommended that the other method of teaching along with frequent reinforcement can be implemented for improving the knowledge regarding prevention of ill effects of television watching on behaviour among school age children.

\section{BIBLIOGRAPHY}

1. Grace Akella, RiaReis, Primary school children's perspectives on common diseases and medicine used. Journal of Health. 2007. Jun; 7(2):65)

2. Ann. Ashworth, Alan Jackson, Focusing on malnutrition management to improve child survival in India Journal of Indian Academy of Paediatrics 2007 June; $44(6) ; 413$
3. Brunner and Suddharth's Text book of medical surgical nursing 11th edition; volume 1; Lippincott; P.P. 1746

4. Joys M. Black, Text Book of Medical surgical nursing, $8^{\text {th }}$ edition; volume 2, Elsevier publication, 2008; PP 1698

5. Wikipedia. Mass media denotes a section of media. Computer and mass media. Libel publication, 2004; 18(5):4 - 8

6. Ms. Joseph Suja, "A study to determine the effect of television watching on children as perceived by their parents."(Unpublished Master of Science in Nursing thesis, Rajiv Gandhi University of health Sciences, Bangalore 1998. 